\title{
Tuneable normal stresses in hyperelastic emulsions
}

\author{
Abdoulaye Fall $\odot,{ }^{1, *}$ Brian P. Tighe $\odot,,^{2, \dagger}$ and Daniel Bonn $\odot^{3, \hbar}$ \\ ${ }^{1}$ Laboratoire Navier (UMR 8205), CNRS, Ecole des Ponts ParisTech, Université Gustave Eiffel, 14-20 Boulevard Isaac Newton, Cité \\ Descartes 77420 Champs sur Marne, France \\ ${ }^{2}$ Process and Energy Laboratory, Delft University of Technology, Leeghwaterstraat 39, 2628 CB Delft, Netherlands \\ ${ }^{3}$ Institute of Physics, University of Amsterdam, Science Park 904, 1098 XH Amsterdam, Netherlands
}

(Received 12 October 2021; accepted 11 February 2022; published 2 March 2022)

\begin{abstract}
We show that foams and emulsions can display a fundamentally different normal response to a simple shear deformation. While foams dilate or push outwards on the shearing surfaces, known as a positive Poynting effect, in emulsions the Poynting effect can have either sign and can be tuned by changing the emulsion properties. We relate the sign of the Poynting effect to the presence of a compressible contact network supported by adhesive contacts. When the concentration of surfactant in the continuous phase is low, the emulsions are nonadhesive and push outward on their shearing surfaces, as do the foams. When the surfactant concentration is increased, the emulsions become adhesive due to depletion interactions, and the Poynting effect changes sign. We argue that the adhesive contact network develops a shear modulus that stiffens in response to dilation, which leads to the negative Poynting effect.
\end{abstract}

DOI: 10.1103/PhysRevResearch.4.013167

\section{INTRODUCTION}

When an elastic solid is sheared, it can expand or contract in the direction perpendicular to the shear plane. If the gap between the shearing surfaces is held fixed, the solid instead pushes or pulls on the surfaces. Both of these phenomena are manifestations of the Poynting effect [1]. While there is no mechanical bound fixing the sign of the effect, most materials expand or develop a compressive stress when sheared. By convention, this is called a positive Poynting effect. However, recently, it has become clear that some materials, such as filamentous biopolymer networks, display a negative Poynting effect - they contract or develop a tensile normal stress [2,3].

This raises the question of what determines the sign of the Poynting effect. Elasticity theory does not offer a simple answer because the effect is intrinsically nonlinear: in isotropic solids, symmetry requires the induced normal stresses and volumetric strains to be proportional to the square of a small shear strain $\gamma$. Here we show an experimental model system in which the sign and amplitude of the Poynting effect change by varying the interactions in the system and can therefore be tuned. We study concentrated emulsions made of small droplets of one simple liquid suspended in another. They exhibit an elastic response at the macroscale due to interfacial tension, which penalizes deviations from a spherical

\footnotetext{
*abdoulaye.fall@cnrs.fr

†b.p.tighe@tudelft.nl

¥d.bonn@uva.nl
}

Published by the American Physical Society under the terms of the Creative Commons Attribution 4.0 International license. Further distribution of this work must maintain attribution to the author(s) and the published article's title, journal citation, and DOI. droplet shape [4]. Starting with Taylor [5], many models have been developed to describe droplet deformation, assuming that the interactions between droplet pairs are purely repulsive [6-11]. Here we show that when droplet-droplet interactions are repulsive, the system dilates or pushes outward when sheared. Its Poynting effect is therefore positive, reminiscent of Reynolds dilatancy in granular materials [12]. However, we also show that when attraction between the drops is introduced in a controllable way, the sign of the Poynting effect can be reversed: emulsions with strong attractions develop a negative Poynting effect. This is our central result, namely, that the sign of the Poynting effect in emulsions can be tuned with an experimentally accessible control parameter.

\section{MATERIALS AND METHODS}

Experiments are performed on a regular oil-in-water emulsion of a $1 \mathrm{~Pa}$ s silicone oil from Sigma Aldrich dispersed in bidistilled water. The emulsions are stabilized by the ionic surfactant sodium dodecyl sulfate (SDS) and were prepared at $24000 \mathrm{rpm}$ with an IKA T18 emulsifier. The surfactant concentration within the aqueous phase is between 0.5 and $8 \mathrm{wt} \%$. In all cases the oil volume fraction is $80 \%[13,14]$. The foam used is a commercial shaving foam (Gillette Foamy Regular). The rheological properties are investigated by a stress-controlled rheometer (Anton Paar MCR 502). The temperature is controlled at $20.0{ }^{\circ} \mathrm{C} \pm 0.1{ }^{\circ} \mathrm{C}$, and a $50 \mathrm{~mm}$ cone and plate geometry (cone angle: $1^{\circ}$ ) are used unless indicated otherwise. All geometries had roughened surfaces in order to prevent wall slip effects during measurements. The measurement of normal stresses in yield stress materials is notoriously difficult because of residual trapped stresses that emerge when loading the material in the rheometer. Here we follow the protocol detailed in [15], where reproducible yield 
stress measurements were made using different methods. Different shear strains are applied to the material, and we measure both the shear stress $\sigma_{x y}$ and the normal stress $F_{Y}$ (thrust per unit cross-sectional area).

\section{RESULTS}

We use aqueous foams as a benchmark for our results in emulsions. Figure 1 shows generic results for a foam and an adhesive (i.e., attractive) emulsion. As the strain increases, the systems behave differently: while a dilatant thrust is observed for the foam, the emulsion pulls inwards on the rheometer head (it "wants" to contract). This qualitative difference between foams and adhesive emulsions is robust to changes in the boundary conditions and shearing geometry. In Fig. 2 we present data for samples sheared between two parallel plates, while the gap between the plates is varied in order to maintain a zero (or very small) thrust. The Supplemental Material [16] provides additional measurements comparing normal stresses in plate-plate and cone-and-plate geometries. In all cases, we observe that while the foam dilates, the adhesive emulsion
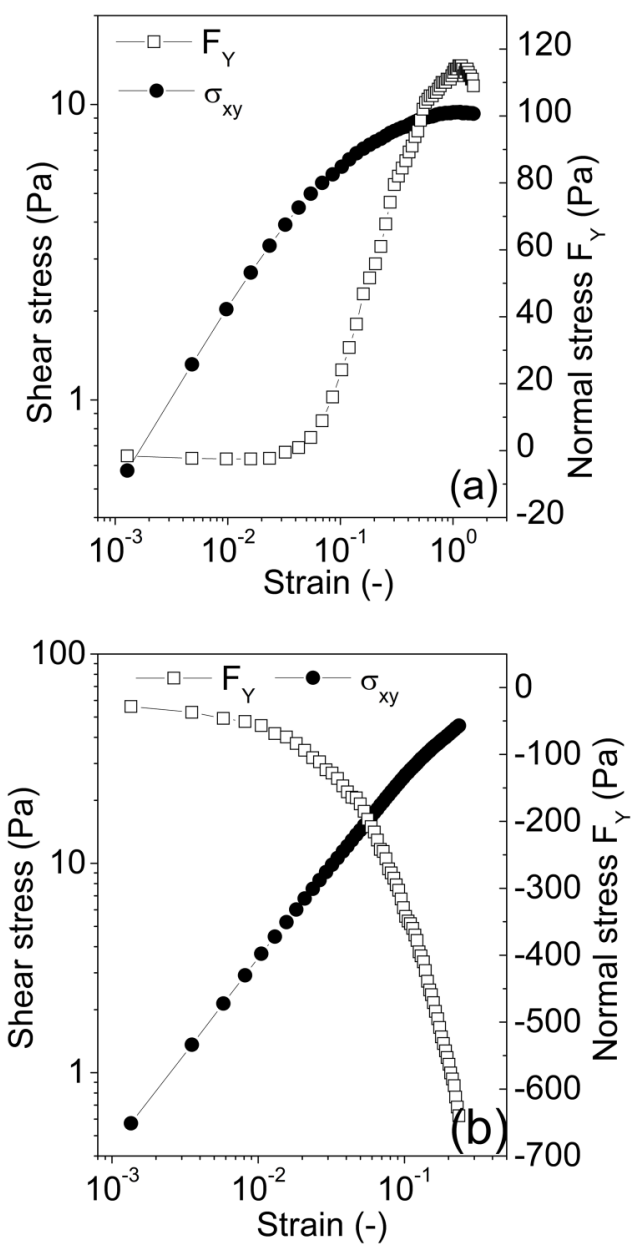

FIG. 1. Normal and shear stresses vs shear strain for (a) a foam and (b) an emulsion with $8 \%$ SDS. Values were continuously measured at a shear rate of $5 \times 10^{-3} \mathrm{~s}^{-1}$ for $600 \mathrm{~s}$. The differential shear modulus is usually defined as the local slope of the stress-strain curve and can thus be read off directly from the figure.

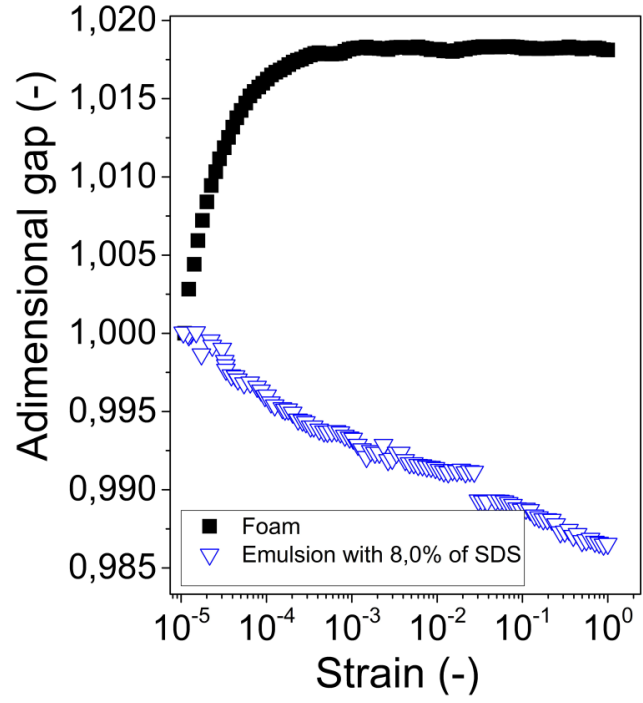

FIG. 2. Evolution of the gap between the shearing plates as a function of shear strain for zero normal stress imposed for foam and emulsion with $8 \%$ SDS. Note that a $50 \mathrm{~mm}$ rough plate-plate geometry is used. Measurements were done during a continuously increasing imposed shear stain: strain levels are imposed for each point waiting $20 \mathrm{~s}$, and then the gap variation is measured averaging over $10 \mathrm{~s}$

contracts, consistent with a positive effect in the foam and a negative effect in the emulsion. Our main goal is to understand the origin of this difference.

Measurements of normal stresses in biopolymer gels $[17,18]$ suggest that compressibility is a prerequisite for a negative Poynting effect. When the gels are sheared rapidly, their water content renders them incompressible, and the Poynting effect is positive. However, water can be expelled from the gels' porous structure if the shear is applied sufficiently slowly. The system is then compressible, and the Poynting effect changes sign. This distinguishes biopolymer networks from, e.g., rubber, which remains incompressible even when sheared slowly.

Inspired by these results, we hypothesize that the negative Poynting effect in adhesive emulsions is due to the formation of a compressible and porous network structure supported by attractive forces between droplets. Short-range attractive forces can be introduced by varying the amount of SDS surfactant in the continuous phase. The micelles exert a depletion interaction on the drops (see, e.g., $[13,19,20]$ ), which leads to an effective attraction between the droplets. Further increasing the SDS concentration increases the micelle concentration, which in turn strengthens the attraction between droplets and leads to flocculation of the droplets. This flocculated system is structurally reminiscent of the biopolymer gels; in particular it can expel water (which is visible at the free surface of the sample) and is therefore compressible.

Two pieces of experimental data provide support for the above scenario. First, we consider the evolution of normal stress as a function of shear strain for emulsions with different SDS concentrations. Figure 3 shows a positive Poynting effect for low surfactant concentrations, reminiscent of foams. As expected, however, we observe a smooth transition between 


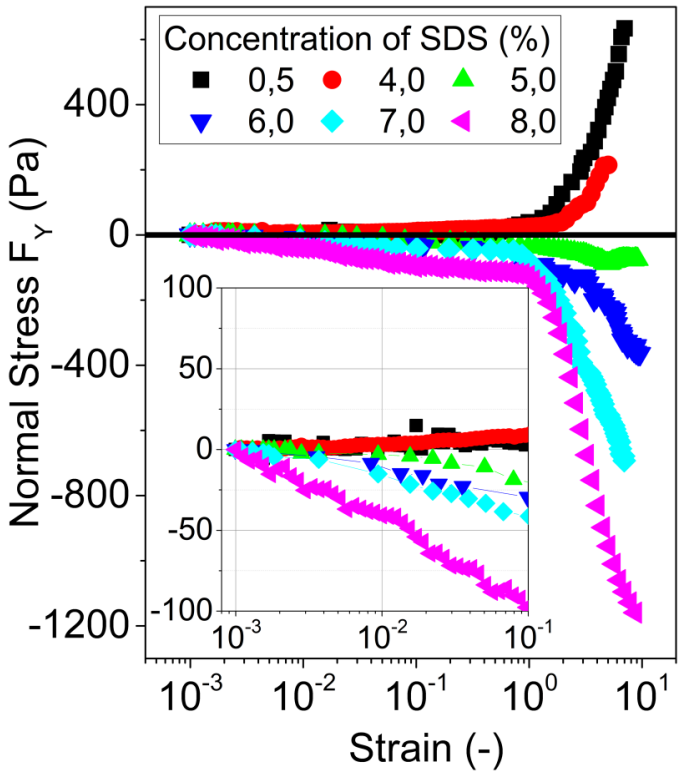

FIG. 3. Normal stresses vs shear strain for emulsions with different concentrations of SDS. The horizontal black line is at $F_{Y}=0$. The inset shows the same data focusing on smaller strains.

positive and negative thrust when increasing the amount of surfactant in the continuous phase.

Second, we seek direct visual evidence of network formation in attractive emulsions by using concentrated samples that have been diluted. Confocal microscopy images show that isolated Brownian droplets are observed for low surfactant concentration, whereas for high concentration the sample is composed of large aggregates (Fig. 4). Since depletion interactions are not sensitive to oil volume fraction [21], we conclude that $0.5 \mathrm{wt} \%$ surfactant leads to a nonadhesive emulsion, whereas 8 wt $\%$ results in an adhesive system $[13,14]$. In repulsive emulsions, droplets repel each other at any center-to-center distance. On the other hand, adhesive emulsions exhibit an attractive potential well at a distance given by the size of the micelles [22]. Consequently, droplets in attractive emulsions flocculate and form persistent gel-like structures, whereas drops in repulsive emulsions do not. It is not possible to give an aggregate size for the attractive emulsions, however, as we necessarily have to dilute the system before the flocculation becomes evident.
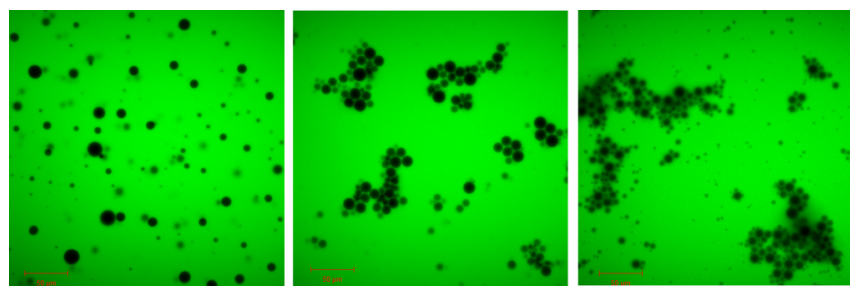

FIG. 4. Confocal imaging of emulsions of silicone oil droplets in water stabilized by SDS. Images were taken after dilution of the concentrated emulsions down to an oil volume fraction of $0.1 \%$ at $40 \times$ magnification. Left: Emulsion with 0.5 wt $\%$ SDS. Middle: Emulsion with 4 wt \% SDS. Right: Emulsion with 8 wt \% SDS. The scale bar in all images is $50 \mu \mathrm{m}$.
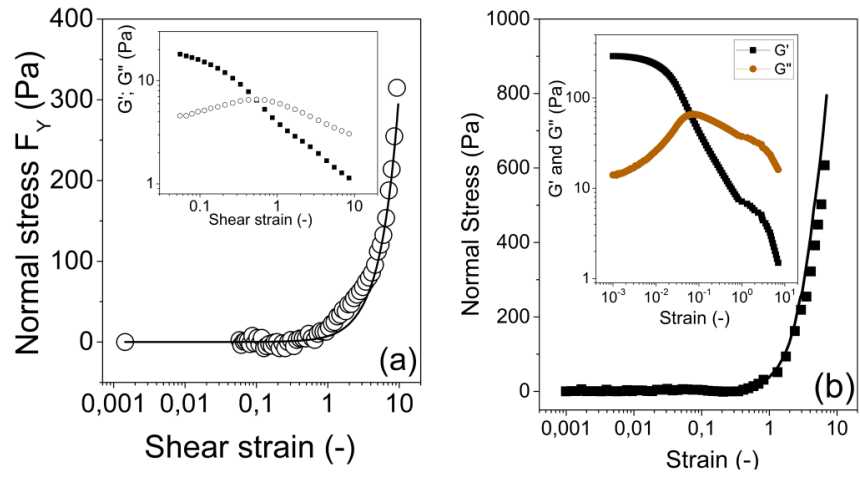

FIG. 5. Normal stresses vs shear strain for (a) the foam and (b) nonadhesive emulsion with $0.5 \%$ SDS. The solid line is $2\left|G^{*}(\omega, \gamma)\right| \gamma^{2}$ (see text). The insets show the results of oscillatory shear measurements at a frequency of $1 \mathrm{~Hz}$ : storage $\left(G^{\prime}\right.$, squares) and loss $\left(G^{\prime \prime}\right.$, circles) modulii as a function of oscillatory strain amplitude.

\section{DISCUSSION}

We now aim to provide a theoretical interpretation of our finding that networks of adhesive emulsion droplets display a negative Poynting effect, unlike repulsive emulsions and foams. We observe that the sign of the normal stress is established immediately upon shearing, when elastic storage dominates loss, and does not change when the loss modulus dominates at larger strains. Therefore, we can understand the sign of the effect by investigating the initial quadratic growth of the normal stress in a hyperelastic model, which neglects dissipation.

As a preliminary, it is useful to recall a textbook result for incompressible hyperelastic solids sheared in cone-plate geometry at fixed gap height. In this case a coincidence of two fundamental relations fully determines the Poynting effect. First, when incompressible media are sheared at fixed gap, the thrust per unit cross sectional area is $F_{Y}=2 N_{1}$, with the first normal stress difference defined as $N_{1}=\sigma_{X X}-\sigma_{Y Y}$ for a shear stress $\sigma_{X Y}$ (see Fig. 6 below) [23]. The second relation concerns hyperelastic solids, i.e., solids that are reversibly elastic for some range of strains extending beyond linear response [24,25]. In these systems $N_{1}=G_{0} \gamma^{2}+O\left(\gamma^{4}\right)$, where $G_{0}$ is the linear elastic shear modulus. Combined, these results require $F_{Y}=2 G_{0} \gamma^{2}$, a positive Poynting effect. Our experimental results for foams and repulsive emulsions compare favorably with this classic result, provided we account for viscoelasticity by replacing $G_{0}$ with $\left|G^{*}(\omega, \gamma)\right|$, where $\omega$ is the shearing frequency and $G^{*}$ is the independently measured complex shear modulus (Fig. 5).

For simplicity, we consider a cube of unit volume containing an isotropic, compressible, and hyperelastic material. As shown in Fig. 6, the cube is deformed such that a material element initially at $\boldsymbol{X}=(X, Y, Z)^{T}$ is located at $\boldsymbol{x}=(X+$ $\left.\gamma Y,\left(1+\xi_{Y}\right) Y, Z\right)^{T}$ after deformation. Here $\gamma$ parameterizes shear, while $\xi_{Y}$ is a dimensionless dilation in the gradient direction. We now ask how the thrust $F_{Y}$ develops as a function of $\gamma$ when $\xi_{Y}=0$ is imposed. Recalling that $F_{Y} \propto \gamma^{2}$ due to symmetry, the Poynting effect is characterized by the 


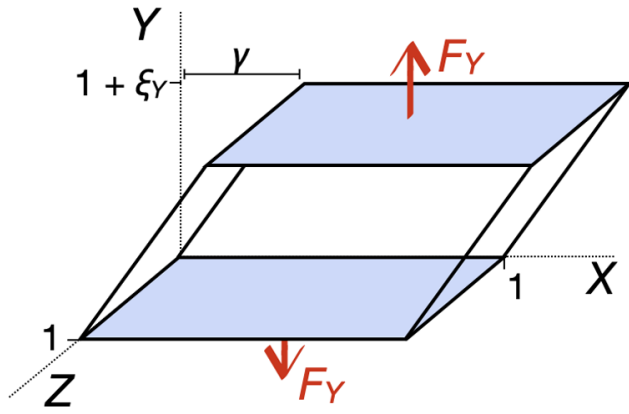

FIG. 6. Coordinates and deformation used in calculating the Poynting coefficient $\chi$.

coefficient

$$
\chi=\left[\left(\frac{\partial^{2} F_{Y}}{\partial \gamma^{2}}\right)_{\xi_{Y}}\right]_{0},
$$

evaluated in the initial condition. This coefficient can be related to properties of the unsheared system by extending the approach developed in Refs. [26-28]. In brief, we write down the total differential of the strain energy density $d W=$ $\left(S_{X Y}+\gamma S_{Y Y}\right) d \gamma+\left(1+\xi_{Y}\right) S_{Y Y} d \gamma$ in terms of elements of the second Piola-Kirchoff stress tensor $\boldsymbol{S}$. We then use the Maxwell relation corresponding to this differential to solve for $\chi$ in terms of (mixed) derivatives of $S_{X Y}$ and $S_{Y Y}$. After some algebra (details are given in the Supplementary Material [16]), we find

$$
\chi=-\left[\left(\frac{\partial G}{\partial \xi_{Y}}\right)\right]_{0},
$$

where $G\left(\xi_{Y}\right)$ is the differential shear modulus evaluated at $\gamma=0$ and arbitrary dilation.

The above result illuminates the role of compressibility in the Poynting effect. The derivative $\left[\partial G / \partial \xi_{Y}\right]_{0}$ measures how much the differential shear modulus changes when the initial condition is stretched. Hence, a negative Poynting effect requires the shear modulus to stiffen in response to stretching. This conclusion is robust to changes in the boundary conditions, e.g., imposing zero stress on the surfaces with normals in the $Y$ and/or $Z$ directions, instead of zero dilation. While the equivalent of Eq. (2) changes, in each case the Poynting effect remains negative when $\left[\partial G / \partial \xi_{Y}\right]_{0}$ is sufficiently large.
There is no bound on mechanical stability that requires $G$ to stiffen or soften under dilation. In repulsive emulsions and foams, the shear modulus is an increasing function of the average number of contacts per droplet [29-32]. Dilation reduces the interfacial area between droplets and eventually causes them to lose contact. The derivative of $G$ is therefore negative, and so the Poynting effect is positive. In contrast, adhesive contacts are "sticky" - once formed, they persist unless some tensile force threshold is exceeded [33-36]. They therefore respond to dilation in a manner akin to biopolymer gels and bead-spring networks, which can support tension without changing their topology. Placing a network under tension increases its shear modulus [27,37,38], yielding a positive derivative $\left[\partial G / \partial \xi_{Y}\right]_{0}$ in Eq. (2). This rationalizes the appearance of a negative Poynting effect with increasing surfactant concentration, as seen in Fig. 3.

\section{CONCLUSION}

The control over the Poynting effect has potential applications, as the difference between a dilatant and contractant normal response impacts the "tackiness" of biomaterials and foodstuffs, of which emulsions are an example. In addition, our considerations here explain a number of sometimes confusing results in the literature. The positive Poynting effect for the repulsive emulsion (and foams) is consistent with theories that assume an incompressible system [39-41] and was indeed already observed previously for foams [41] and concentrated, nonadhesive emulsions. However, for the emulsions the situation was far from being clear: in one set of data normal and shear stresses in steady shear flow were proportional [42], whereas in the other a quadratic dependence was reported for oscillatory shear [15], and in a third publication, a negative Poynting effect was reported but without any explanation [22]. Here we show that the behavior on strain should be quadratic and that the negative normal stress in a different system is likely due to attractive interactions between the drops. Such negative normal stresses were previously reported in biopolymer gels [2,17,18], nanotube suspensions [43], and bead-spring networks [28]. The common denominator between these systems is that they form connected networks, in line with the arguments presented here.

\section{ACKNOWLEDGMENT}

We thank NWO for the financial support of the present research work.
[1] J. H. Poynting, On pressure perpendicular to the shear planes in finite pure shears, and on the lengthening of loaded wires when twisted, Proc. R. S. London. Ser. A 82, 546 (1909).

[2] P. A. Janmey, M. E. McCormick, S. Rammensee, J. L. Leight, P. C. Georges, and F. C. MacKintosh, Negative normal stress in semiflexible biopolymer gels, Nat. Mater. 6, 48 (2007).

[3] E. Conti and F. C. MacKintosh, Cross-Linked Networks of Stiff Filaments Exhibit Negative Normal Stress, Phys. Rev. Lett. 102, 088102 (2009).
[4] C. L. Tucker III and P. Moldenaers, Microstructural evolution in polymer blends, Annu. Rev. Fluid Mech. 34, 177 (2002).

[5] G. I. Taylor, The formation of emulsions in definable fields of flow, Proc. R. Soc. London, Ser. A 146, 501 (1934).

[6] J. Rallison, The deformation of small viscous drops and bubbles in shear flows, Annu. Rev. Fluid Mech. 16, 45 (1984).

[7] H. A. Stone, Dynamics of drop deformation and breakup in viscous fluids, Annu. Rev. Fluid Mech. 26, 65 (1994). 
[8] P. L. Maffettone and F. Greco, Ellipsoidal drop model for single drop dynamics with non-Newtonian fluids, J. Rheol. 48, 83 (2004).

[9] G. Batchelor, The stress generated in a non-dilute suspension of elongated particles by pure straining motion, J. Fluid Mech. 46, 813 (1971).

[10] D. J. Durian, Foam Mechanics at the Bubble Scale, Phys. Rev. Lett. 75, 4780 (1995).

[11] B. P. Tighe, Relaxations and Rheology Near Jamming, Phys. Rev. Lett. 107, 158303 (2011).

[12] J. Ren, J. A. Dijksman, and R. P. Behringer, Reynolds Pressure and Relaxation in a Sheared Granular System, Phys. Rev. Lett. 110, 018302 (2013).

[13] L. Bécu, S. Manneville, and A. Colin, Yielding and Flow in Adhesive and Nonadhesive Concentrated Emulsions, Phys. Rev. Lett. 96, 138302 (2006).

[14] A. Fall, J. Paredes, and D. Bonn, Yielding and Shear Banding in Soft Glassy Materials, Phys. Rev. Lett. 105, 225502 (2010).

[15] H. de Cagny, M. Fazilati, M. Habibi, M. M. Denn, and D. Bonn, The yield normal stress, J. Rheol. 63, 285 (2019).

[16] See Supplemental Material at http://link.aps.org/supplemental/ 10.1103/PhysRevResearch.4.013167 for details supporting information to accompany the main text, additional data for the comparison between measurements done in cone-plate and plate-plate geometries, more details on the theoretical calculation of the Poynting coefficient in hyperelastic solids.

[17] H. C. G. de Cagny, B. E. Vos, M. Vahabi, N. A. Kurniawan, M. Doi, G. H. Koenderink, F. C. MacKintosh, and D. Bonn, Porosity Governs Normal Stresses in Polymer Gels, Phys. Rev. Lett. 117, 217802 (2016).

[18] M. Vahabi, B. E. Vos, H. C. G. de Cagny, D. Bonn, G. H. Koenderink, and F. C. MacKintosh, Normal stresses in semiflexible polymer hydrogels, Phys. Rev. E 97, 032418 (2018).

[19] M. P. Aronson, The role of free surfactant in destabilizing oilin-water emulsions, Langmuir 5, 494 (1989).

[20] S. S. Datta, D. D. Gerrard, T. S. Rhodes, T. G. Mason, and D. A. Weitz, Rheology of attractive emulsions, Phys. Rev. E 84, 041404 (2011).

[21] J. Bibette, D. Roux, and F. Nallet, Depletion Interactions and Fluid-Solid Equilibrium in Emulsions, Phys. Rev. Lett. 65, 2470 (1990).

[22] A. Montesi, A. A. Pena, and M. Pasquali, Vorticity Alignment and Negative Normal Stresses in Sheared Attractive Emulsions, Phys. Rev. Lett. 92, 058303 (2004).

[23] C. W. Macosko, Rheology: Principles, Measurements, and Applications (Wiley-VCH, New York, 1994).

[24] R. W. Ogden, Non-linear Elastic Deformations (Dover, Mineola, NY, 1984).

[25] H. Xiao and L. H. He, A unified exact analysis for the Poynting effects of cylindrical tubes made of Hill's class of Hookean compressible elastic materials at finite strain, Int. J. Solids Struct. 44, 718 (2007).
[26] D. Weaire and S. Hutzler, Dilatancy in liquid foams, Philos. Mag. 83, 2747 (2003).

[27] B. P. Tighe, Shear dilatancy in marginal solids, Granular Matter 16, 203 (2014).

[28] K. Baumgarten and B. P. Tighe, Normal Stresses, Contraction, and Stiffening in Sheared Elastic Networks, Phys. Rev. Lett. 120, 148004 (2018).

[29] A. Saint-Jalmes and D. Durian, Vanishing elasticity for wet foams: Equivalence with emulsions and role of polydispersity, J. Rheol. 43, 1411 (1999).

[30] G. Katgert and M. van Hecke, Jamming and geometry of twodimensional foams, Europhys. Lett. 92, 34002 (2010).

[31] K. W. Desmond, P. J. Young, D. Chen, and E. R. Weeks, Experimental study of forces between quasi-two-dimensional emulsion droplets near jamming, Soft Matter 9, 3424 (2013).

[32] I. Jorjadze, L.-L. Pontani, and J. Brujic, Microscopic Approach to the Nonlinear Elasticity of Compressed Emulsions, Phys. Rev. Lett. 110, 048302 (2013).

[33] G. Lois, J. Blawzdziewicz, and C. S. O'Hern, Jamming Transition and New Percolation Universality Classes in Particulate Systems with Attraction, Phys. Rev. Lett. 100, 028001 (2008).

[34] I. Jorjadze, L.-L. Pontani, K. A. Newhall, and J. Brujić, Attractive emulsion droplets probe the phase diagram of jammed granular matter, Proc. Natl. Acad. Sci. 108, 4286 (2011).

[35] D. J. Koeze and B. P. Tighe, Sticky Matters: Jamming and Rigid Cluster Statistics with Attractive Particle Interactions, Phys. Rev. Lett. 121, 188002 (2018).

[36] D. J. Koeze, L. Hong, A. Kumar, and B. P. Tighe, Elasticity of jammed packings of sticky disks, Phys. Rev. Res. 2, 032047(R) (2020).

[37] B. E. Vos, L. C. Liebrand, M. Vahabi, A. Biebricher, G. J. Wuite, E. J. Peterman, N. A. Kurniawan, F. C. MacKintosh, and G. H. Koenderink, Programming the mechanics of cohesive fiber networks by compression, Soft Matter 13, 8886 (2017).

[38] M. Merkel, K. Baumgarten, B. P. Tighe, and M. L. Manning, A minimal-length approach unifies rigidity in underconstrained materials, Proc. Natl. Acad. Sci. USA 116, 6560 (2019).

[39] R. Larson, The elastic stress in "film fluids", J. Rheol. 41, 365 (1997).

[40] R. G. Larson, The Structure and Rheology of Complex Fluids, Topics in Chemical Engineering Vol. 150 (Oxford University Press, New York, 1999), Chap. 9, pp. 388-440.

[41] V. Labiausse, R. Höhler, and S. Cohen-Addad, Shear induced normal stress differences in aqueous foams, J. Rheol. 51, 479 (2007).

[42] J. R. Seth, L. Mohan, C. Locatelli-Champagne, M. Cloitre, and R. T. Bonnecaze, A micromechanical model to predict the flow of soft particle glasses, Nat. Mater. 10, 838 (2011).

[43] S. Lin-Gibson, J. A. Pathak, E. A. Grulke, H. Wang, and E. K. Hobbie, Elastic Flow Instability in Nanotube Suspensions, Phys. Rev. Lett. 92, 048302 (2004). 